\title{
Low-dose radiation therapy for massive chylous leakage after subtotal gastrectomy
}

\author{
Sang-Won Kim, MD, Jung Hoon Kim, MD \\ Department of Radiation Oncology, Konyang University College of Medicine, Daejeon, Korea
}

\begin{abstract}
Massive chylous leakage is a rare postoperative complication that can prolong hospital stay and cause secondary morbidities. Conservative management strategies are the treatment of choice; however, radiation therapy (RT) can be used as an alternative for cases that are refractory to conservative treatment. Herein, we report a 69-year-old female patient who suffered from massive chylous leakage after subtotal gastrectomy. Due to persistent massive chylous leakage, she was scheduled to undergo low-dose RT. Radiation was delivered with a daily dose of $1 \mathrm{~Gy}$, using an anterior-posterior and posterior-anterior beam arrangement. The clinical target volume encompassed the entire lymph node area of the D2 dissection. RT was completed at the total dose of 8 Gy because the amount of chylous leakage declined rapidly. Percutaneous drainage tube was removed after 3 days of RT. The patient did not complain of any symptoms related to massive chylous leakage 2 years after the completion of RT.
\end{abstract}

Keywords: Chylous ascites, Radiotherapy, Gastrectomy

\section{Introduction}

Chyle, originating from the Greek word for juice, is a milky body fluid consisting of lymph and triglyceride fat in a stable emulsion. Chyle is formed in the small intestine during digestion and drained into the junction of the left subclavian and jugular vein via the thoracic duct.

Chylous leakage can occur secondary to lymphoma, infectious disease, congenital abnormalities and cardiothoracic or abdominal surgery [1]. Chylous leakage may manifest as chylothorax, chylopericardium, or chylous ascites depending upon the involved site. Although the incidence of chylous leakage is low, its development can negatively influence patients by prolonging the length of hospitalization, increasing the risk of secondary morbidities, and decreasing quality of life.
Various conservative management strategies including low fat diet, total parenteral nutrition and administration of somatostatin (or its analogues) are available [1]. In most cases, conservative management is sufficient to control massive chylous leakage. However, a small subset of patients who develop massive chylous leakage do not respond to conservative management. Surgical ligation or peritoneovenous shunts are options for refractory chylous leakage; however, they are associated with a high risk for complications. Radiation therapy (RT) is a lesser-known treatment for massive chylous leakage that is refractory to conservative management. Several authors, primarily from Germany, have reported the possible role of RT as an effective treatment for refractory chylous leakage [2-7].

We present a 69-year-old female patient who received successful low-dose RT to treat massive chylous leakage that

Received 04 April 2017, Revised 12 April 2017, Accepted 13 April 2017.

Correspondence: Jung Hoon Kim, MD, Department of Radiation Oncology, Konyang University College of Medicine, 158 Gwanjeodong-ro, Seo-gu, Daejeon 35365, Korea. Tel: +82-42-600-6761, Fax: +82-42-600-9469, E-mail: medduck@kyuh.ac.kr

(c) This is an Open Access article distributed under the terms of the Creative Commons Attribution Non-Commercial License (http://creativecommons.org/ licenses/by-nc/4.0/) which permits unrestricted non-commercial use, distribution, and reproduction in any medium, provided the original work is properly cited.

www.e-roj.org 
developed after subtotal gastrectomy for early gastric cancer.

\section{Case Report}

At age 69, a female patient had a regular medical examination that included an upper gastrointestinal endoscopy. On

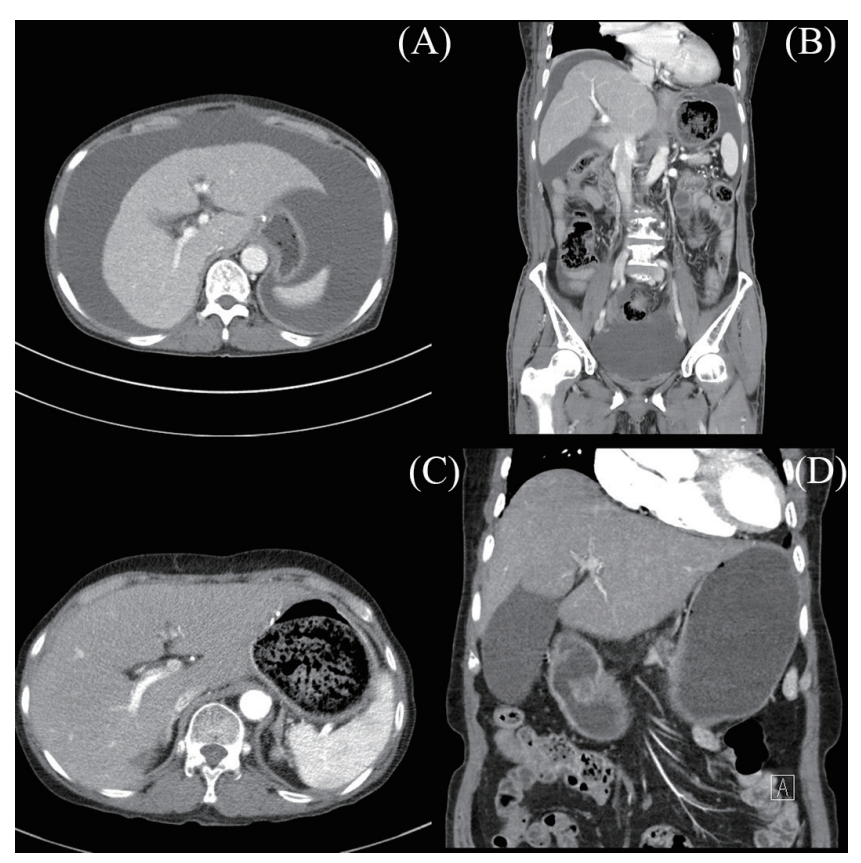

Fig. 1. Axial and coronal images of computed tomography with contrast enhancement showing massive chylous leakage before radiation therapy $(A, B)$ and disappearance of chylous leakage 1 month after completion of radiation therapy $(C, D)$. endoscopy, a 4-cm-sized fungating mass with irregular margins was found at the posterior wall of the antrum. Biopsy confirmed a poorly differentiated adenocarcinoma. Imaging work-ups revealed a primary tumor confined to the stomach without regional or distant metastasis. She underwent subtotal gastrectomy with D2 lymph node dissection. The intraoperative course was uneventful, with an estimated blood loss of 100 $\mathrm{mL}$. She was discharged 3 weeks after surgery and did not complain of any discomfort during her stay at the hospital.

A computed tomography (CT) scan performed 3 months after surgery showed a large amount of ascites in the abdominal cavity (Fig. 1A and 1B). She complained of persistent abdominal distension and administration of diuretics for 1 month did not resolve massive chylous leakage. From the next month, she underwent monthly paracentesis with diuretics. Examination of the body fluid from paracentesis showed milky appearance and revealed a high level of protein $(4,524.40 \mathrm{mg} / \mathrm{dL})$. There were no clinical signs of infection. She was also recommended to have a low-fat diet by her physician.

Despite monthly paracentesis for 5 months, the volume of drained fluid did not decline and the median amount of drainage was $3,000 \mathrm{~mL}$ (range, 1,800 to $4,500 \mathrm{~mL}$ ) (Fig. 2). Five months after surgery, she was readmitted and received a percutaneous drainage tube insertion. During the first hospitalization, the total amount of drainage fluid was 16,070 $\mathrm{mL}$ over 9 days. One week after being discharged, she was hospitalized again because of persistent chyle leakage (median, $700 \mathrm{~mL} /$ day). Although lymphoscintigraphy was performed, the leakage site was not found and the plan for surgical ligation was abandoned. The amount of chylous leakage did not reduce

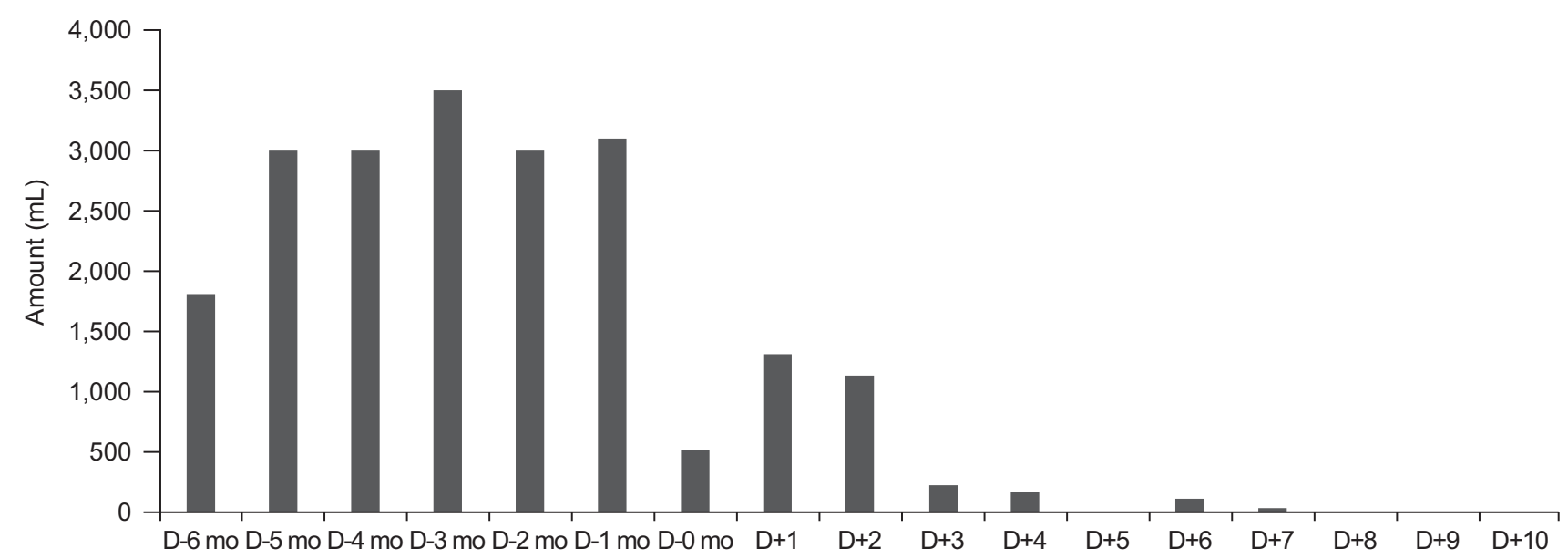

Fig. 2. Graph indicating the amount of chylous leakage from paracentesis or percutaneous drainage tube before, during, and after radiation therapy. 


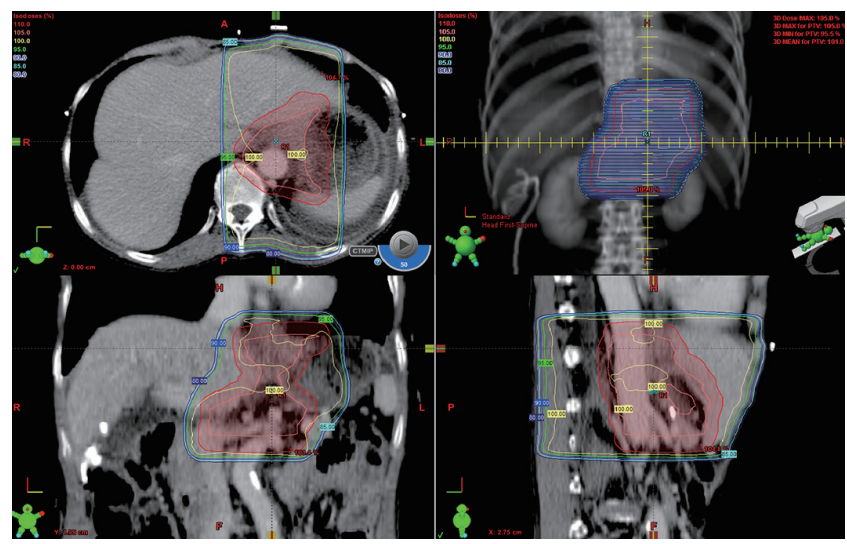

Fig. 3. Radiation therapy plan showing isodose line on axial, sagittal, and coronal images. Anterior-posterior and posterioranterior portal was used.

until the second week of hospitalization and the patient was consulted for low-dose RT.

Informed consent was obtained from the patient before initiation of RT. A planning CT was scanned with free breathing. The clinical target volume encompassed the entire lymph node station of the D2 dissection (Fig. 3). A 1-cm margin was added to the planning target volume. A daily dose of $1 \mathrm{~Gy}$ with a dose-rate of $600 \mathrm{MU} / \mathrm{min}$ was irradiated, using an anterior-posterior/posterior-anterior beam arrangement. On day 4 of radiotherapy, the amount of chylous leakage was below $210 \mathrm{~mL}$ and had totally disappeared by the time the dose reached $8 \mathrm{~Gy}$. During RT, she did not present any acute side effects. Percutaneous drainage tube was removed 3 days after completion of RT.

Two years after the completion of RT, ascites were not identified on serial follow-up CT examinations (Fig. 1C and 1D). Any late toxicities related to RT were not observed until the last follow-up.

\section{Discussion}

Similar to previous studies, this study showed successful RT for massive chylous leakage as a postoperative complication [27]. A total radiation dose of $8 \mathrm{~Gy}$ in 8 fractions was sufficient to control the massive chylous leakage. In addition, the patient did not complain about any treatment related acute or late toxicities.

The incidence of massive chylous leakage as a postoperative complication varies according to the site of surgery and is $0.3 \%-0.6 \%$ for gastrectomy with D2 dissection [1]. Since massive chylous leakage causes morbidities including prolongation of hospitalization, infection and malnutrition (that were also observed in the present case), proper management is important for recovery of the patient and improvement in quality of life. The first treatment option for massive chylous leakage after major abdominal surgery is a conservative management strategy that includes a low-fat diet with supplementation of medium chain triglycerides or total parenteral nutrition. Conservative management is expected to resolve massive chylous leakage in $23 \%$ to $100 \%$ of patients $[1,6]$.

The addition of somatostatin or its analogues can decrease the period of conservative management [8]. Somatostatin reduces massive chylous leakage by decreasing lymph flow in the lymphatic vessels, which is achieved by reduction of fat absorption in the bowel and lowering the level of triglycerides in the thoracic duct [9]. Some authors suggest routine use of somatostatin or its analogues for postoperative chylous leakage because they can decrease complications after radical surgery and have lower side effects [10]. However, the benefit of somatostatin and its analogues are established only in the pancreatic surgery $[8,10]$.

The median duration of conservative management strategies for resolution of chylous leakage ranged from 3.5 to 29 days [1]. The adequate time to assess the efficacy of conservative treatments is 6 to 8 weeks [5]. Surgical or interventional approaches such as ligation or peritoneovenous shunts can be considered for massive chylous leakage that is refractory to conservative management strategies, generally defined as the total amount of drainage fluid $>500 \mathrm{~mL} /$ day. However, surgical ligation can be used only when lymphoscintigraphy has identified the leakage site. Furthermore, peritoneovenous shunts are accompanied by a high rate of complications including sepsis, disseminated intravascular coagulation, electrolyte imbalance, and air embolism [11]. Due to these shortcomings, surgical or interventional approaches should be considered with caution.

In contrast, RT is not widely implemented for the treatment of massive chylous leakage that is refractory to conservative management strategies. Several systematic reviews did not incorporate RT as one of the treatment options for massive chylous leakage $[1,11]$. Since only a few articles report the effects of RT on massive chylous leakage and most of them are case reports or small retrospective studies [2-7], lack of evidence is the main cause for the unpopularity of RT (Table 1).

Nevertheless, all published studies showed feasibility of RT with a higher success rate and fewer treatment-related side effects. Neu et al. [2] reported that 27 out of 28 evaluable 
patients with Iymphatic fistula or lymphocele achieved complete remission after RT with a total dose of 3-12 Gy. Mayer et al. [4] investigated outcomes of 17 patients with 19 fistulas who received low-dose RT with a total dose of 1-12 Gy. Fifteen fistulas were successfully obliterated after low-dose RT.

Along with other previous studies [2-7], our study indicate that massive chylous leakage after radical surgery can be resolved by low dose RT with small fraction size. Furthermore, due to low total radiation dose and small fraction size, acute and late side effects were not observed. Another notable finding in some previous studies was early termination of RT before delivery of the planned dose in a considerable nu mber of cases due to the rapid decline of the chylous leakage volume $[2,7]$. Considering that the risk of acute and late complications of RT increases as the total radiation dose increases, daily evaluation of treatment response should be required for delivery of radiation dose as low as reasonably achievable. In this case report, the amount of massive chylous leakage was nearly $0 \mathrm{~mL}$ by the end of RT with a total dose of $8 \mathrm{~Gy}$. We were able to remove the percutaneous drainage tube on the 3rd day after completion of RT.

Based on these findings, RT can be a feasible treatment option for massive chylous leakage that is refractory to conservative management strategies. Furthermore, RT has advantages over second surgery or interventions because it can avoid several complications associated with invasive procedures and is not restricted by the results of lymphoscintigraphy.

RT can also control massive chylous leakage related to Iymphoma. A couple of case reports demonstrated that massive chylous leakage disappeared after RT with total dose ranging from 12-36 Gy in fraction size of 1-1.8 Gy, regardless of progression of lymphoma [12,13]. Unfortunately, no available evidence exists regarding the effects of RT for massive chylous leakage from other causes.

Radiobiological explanations for improvement of massive chylous leakage are not fully understood. Based on previous literature, it can be deduced that rather than cell killing, low-dose radiation exerts an influence on modulation of inflammatory reaction, thus changing endothelial cell adhesion properties and cellular functions in the lymphatic vessel [4].

In summary, low-dose RT is an effective treatment for massive chylous leakage after radical surgery, which is refractory to conservative management. Treatment is well tolerated without any acute or late complications since low total radiation dose with small fraction size is sufficient to control massive chylous leakage. Considering advantages of RT over surgical or interventional approaches and its feasibility, we suggest that RT should be a preferred treatment option for massive chylous leakage that is refractory to conservative management. Because the amount of chylous leakage can decline rapidly within a couple of days after initiation of RT, daily monitoring of treatment efficacy should be accompanied to achieve delivery of the lowest possible total radiation dose.

\section{Conflict of Interest}

No potential conflict of interest relevant to this article was reported.

\section{References}

1. Weniger M, D'Haese JG, Angele MK, Kleespies A, Werner J, Hartwig W. Treatment options for chylous ascites after major abdominal surgery: a systematic review. Am J Surg

Table 1. Literatures reporting the effect of radiation therapy on massive chylous leakage after major surgery

\begin{tabular}{lccccc} 
& Year & No. of cases & Total RT dose (Gy) & Fx size (Gy) & Success rate (\%) \\
\hline Neu et al. [2] & & 29 & $3-12$ & 1 & 96.4 \\
Dietl et al. [3] & 2000 & 28 & $3-15$ & $3-5$ & 100 \\
Mayer et al. [4] & 2005 & 17 (19 fistulas) & $1-12$ & $0.3-2$ & 78.9 \\
Sziklavari et al. [6] & 2005 & 7 & Mean 8.5 & $1-1.5$ & 1 \\
Perez et al. [5] & 2013 & 1 & 15 & 1 & 100 \\
Corradini et al. [7] & 2013 & 1 & 8 & 1 & 100 \\
This study & 2015 & 1 & 8 & & 94.2 \\
Total & & & & & 9 \\
\hline
\end{tabular}

RT, radiation therapy; Fx, fraction.

a) Of 28 evaluable patients for treatment response, 27 patients showed a complete remission. 
2016;211:206-13.

2. Neu B, Gauss G, Haase W, Dentz J, Husfeldt KJ. Radiotherapy of Iymphatic fistula and Iymphocele. Strahlenther Onkol 2000;176:9-15.

3. Dietl B, Pfister K, Aufschlager C, Kasprzak PM. [Radiotherapy of inguinal lymphorrhea after vascular surgery: a retrospective analysis]. Strahlenther Onkol 2005;181:396-400.

4. Mayer R, Sminia P, McBride WH, et al. Lymphatic fistulas: obliteration by low-dose radiotherapy. Strahlenther Onkol 2005;181:660-4.

5. Perez J, Arribas JM, Cardenas E, Gutierrez F, Taboada $\mathrm{R}$, Cassinello N. Radiotherapeutic management of the chyloperitoneum following abdominal aortic aneurysm repair. J Vasc Surg Venous Lymphat Disord 2013;1:409-11.

6. Sziklavari Z, Allgauer M, Hubner G, et al. Radiotherapy in the treatment of postoperative chylothorax. J Cardiothorac Surg 2013;8:72.

7. Corradini S, Liebig S, Niemoeller OM, Zwicker F, Lamade W. Successful radiation treatment of chylous ascites following pancreaticoduodenectomy. Strahlenther Onkol 2015;191:448-
52.

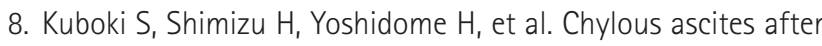
hepatopancreatobiliary surgery. Br J Surg 2013;100:522-7.

9. Collard JM, Laterre PF, Boemer F, Reynaert M, Ponlot R. Conservative treatment of postsurgical lymphatic leaks with somatostatin-14. Chest 2000;117:902-5.

10. Connor S, Alexakis N, Garden OJ, Leandros E, Bramis J, Wigmore SJ. Meta-analysis of the value of somatostatin and its analogues in reducing complications associated with pancreatic surgery. Br J Surg 2005;92:1059-67.

11. Aalami 00, Allen DB, Organ CH. Chylous ascites: a collective review. Surgery 2000;128:761-78.

12. Gerstein J, Kofahl-Krause D, Fruhauf J, Bremer M. Complete remission of a lymphoma-associated chylothorax by radiotherapy of the celiac trunk and thoracic duct. Strahlenther Onkol 2008;184:484-7.

13. Laila K, Klaus $H, H o A D$, Jurgen $D$, Mathias WH. Successful abdominal irradiation in two patients with therapy-resistant chylous ascites due to follicular lymphoma. Ann Hematol 2016;95:1563-5. 